ing the range of known Bernoullian numbers, calculated $\gamma$ to 263 place of decimals.

Inasmuch as Legendre's table has not often been reprinted, it may be of interest to give the results of my computation to the eleventh place of decimals. They are as follows :

$$
\text { Values of } s_{n}=\frac{1}{2^{n}}+\frac{1}{3^{n}}+\frac{1}{4^{n}}+\cdots
$$

\begin{tabular}{rrrrrrrr}
$n$ & \multicolumn{2}{c}{$s_{n}$} & & \multicolumn{4}{c}{$s_{n}$} \\
2 & .64493 & 40668 & 5 & 14 & .00006 & 12481 & 4 \\
3 & .20205 & 69031 & 6 & 15 & 3 & 05882 & 4 \\
4 & 8232 & 32337 & 1 & 16 & 1 & 52822 & 6 \\
5 & 3692 & 77551 & 4 & 17 & 76372 & 0 \\
6 & 1734 & 30619 & 8 & 18 & 38172 & 9 \\
7 & 834 & 92773 & 8 & 19 & 19082 & 1 \\
8 & 407 & 73562 & 0 & 20 & 9539 & 6 \\
9 & 200 & 83928 & 3 & 21 & 4769 & 3 \\
10 & 99 & 45751 & 3 & 22 & 2384 & 5 \\
11 & 49 & 41886 & 0 & 23 & 1192 & 2 \\
12 & 24 & 60865 & 5 & 24 & 596 & 1 \\
13 & 12 & 27133 & 5 & 25 & 298 & 0
\end{tabular}

[The values for $n>25$ are obtained each by dividing its predecessor by 2.]

$$
\begin{gathered}
\text { AnNAPOLIS, } \\
\text { May, } 1906 .
\end{gathered}
$$

\title{
ON CERTAIN PROPERTIES OF WRONSKIANS AND RELATED MATRICES.
}

BY PROFESSOR D. R. CURTISS.

(Read before the Chicago Section of the American Mathematical Society, A pril 14, 1906.)

In this note I shall present theorems of a very general character on the vanishing of Wronskians and related matrices. Proofs, however, will be reserved for subsequent publication in more extended form.

Let $u_{1}, u_{2}, \cdots, u_{n}$ be functions, real or complex, of the real variable $x$, having finite derivatives of the first $k$ orders 
$(k \geqq n-1)$ at each point of an interval $I$. This interval may be finite or infinite, and, if limited in either direction, may or may not include its end points. We shall use the symbol $M_{k}\left(u_{1}, u_{2}, \cdots, u_{n}\right)$ to designate the matrix

$$
\left|\begin{array}{cccc}
u_{1} & u_{2} & \ldots & u_{n} \\
u_{1}^{\prime} & u_{2}^{\prime} & \ldots & u_{n}^{\prime} \\
\cdot & \cdot & \ldots & \cdot \\
\cdot & \cdot & \cdots & \cdot \\
\cdot & \cdot & \cdots & \cdot \\
u_{1}^{(k)} & u_{2}^{(k)} & \ldots & u_{n}^{(k)}
\end{array}\right|
$$

The Wronskian $W\left(u_{1}, u_{2}, \cdots, u_{n}\right)$ will then be the $n$-rowed determinant whose matrix is $M_{n-1}\left(u_{1}, u_{2}, \cdots, u_{n}\right)$.

The first three of the following theorems concern the vanishing of the Wronskian in an infinite set of points belonging to the interval $I$, and having a limiting point $p$ which is also a point of $I$. The symbol $[P]$ will be used to designate such a set when it includes the point $p$. A set which does not contain the limiting point $p$ will be referred to as a set $\left[P^{*}\right]$.

TheORem I. Let $u_{1}, u_{2}, \cdots, u_{n}$ be functions of $x$ which at every point of I have finite derivatives of the first $n$ orders; then if $W\left(u_{1}, u_{2}, \cdots, u_{n}\right)$ vanishes in a point set $[P]$ at least one of the Wronskians $W\left(u_{1}^{\prime}, u_{2}^{\prime}, \cdots, u_{\nu}^{\prime}\right)(\nu=1,2, \cdots, n)$ vanishes in $a \operatorname{set}\left[P^{*}\right]$.

TheOREM II. Let $u_{1}, u_{2}, \cdots, u_{n}$ be functions of $x$ which at every point of I have continuous derivatives of the first $k$ orders $(k \geqq n)$; then if $W\left(u_{1}, u_{2}, \cdots, u_{n}\right)$ vanishes in a point set $[P]$, all the n-rowed determinants of the matrix $M_{l_{i}}\left(u_{1}, u_{2}, \ldots, u_{n}\right)$ vanish at the point $p$.

In this last theorem the functions $u_{1}^{(k)}, u_{2}^{(k)}, \ldots, u_{n}^{(k)}$ are supposed continuous. The following theorem does not make this hypothesis :

THEOREM III. Let $u_{1}^{(k)}, u_{2}^{(k)}, \ldots, u_{n}^{(k)}(k \geqq n)$ exist and be finite at every point of $I$; then if $W\left(u_{1}, u_{2} \cdots, u_{n}\right)$ and its first $k-n+1$ derivatives vanish simultaneously in a point set $[P]$ all the n-rowed determinants of the matrix $M_{k}\left(u_{1}, u_{2}, \cdots, u_{n}\right)$ vanish simultaneously either at $p$ or in a set $\left[P^{*}\right]$. 
From these three theorems we deduce the following, which is easily seen to be a direct consequence of Theorem II when $u_{1}^{(k)}, u_{2}^{(k)}, \ldots, u_{n}^{\left(k_{k}\right)}$ are continuous :

Theorem IV. Let $u_{1}^{(k)}, u_{2}^{(k)}, \ldots, u_{n}^{(k)}(k \geqq n)$ exist and be finite at every point of $I$; then if $W\left(u_{1}, u_{2}, \ldots, u_{n}\right)$ vanishes identically throughout $I$ every n-rowed determinant of $M_{k_{s}}\left(u_{1}, u_{2}, \ldots, u_{n}\right)$ vanishes identically throughout $I$.

For an interval where $W\left(u_{1}, u_{2}, \cdots, u_{n-1}\right)$ does not vanish, Theorem IV has been stated and proved by Bôcher *; this restriction is not necessary to my proof. As a corollary we obtain Bôcher's theorem that if $W\left(u_{1}, u_{2}, \cdots, u_{n}\right) \equiv 0$ then $W\left(u_{1}, u_{2}, \cdots, u_{n+1}\right) \equiv 0$, but Bôcher's assumption of the continuity of $u_{1}^{(n)}, u_{2}^{(n)}, \ldots, u_{n+1}^{(n)}$ is now clearly seen to be unnecessary.

The preceding theorems have an important application in the theory of linear dependence. The results which I have obtained are summarized in the following theorem where the phrase, " $M_{k_{k}}\left(u_{1}, u_{2}, \cdots, u_{n}\right)$ is of constant rank $m<n$ in $I$," means that every $(m+1)$-rowed determinant of this matrix vanishes identically throughout $I$ while the $m$-rowed determinants do not all vanish at any point of $I$ :

Theorem V. If $M_{k}\left(u_{1}, u_{2}, \ldots, u_{n}\right)$ is of constant rank $m<n$ in $I, u_{1}, u_{2}, \ldots, u_{n}$ are linearly dependent and the number of independent linear relations between these functions is $n-m$.

Since $M_{2}\left(u_{1}, u_{2}, \ldots, u_{n}\right)$ is of constant rank if $u_{1}, u_{2}, \ldots, u_{n}$ are solutions of a linear differential equation of order $k$, it is evident that Theorem $\mathrm{V}$ is of wide application. In fact it includes all sufficient conditions for the linear dependence of nonanalytic functions that have been given by Peano and Bôcher, but applies to no cases which do not come under Bôcher's Theorem VI. $\dagger$ This theorem states that if $W\left(u_{1}, u_{2}, \ldots, u_{n}\right) \equiv 0$, while no function (other than zero) of the form

$$
g_{1} u_{1}+g_{2} u_{2}+\cdots+g_{n} u_{n}
$$

(the $g$ 's being constants) vanishes together with its first $k$ deriva tives at any point of $\mathrm{I}$, the functions $u_{1}, u_{2}, \ldots, u_{n}$ are linearly

\footnotetext{
* Transactions Amer. Math. Society, vol. 2 (1901), p. 139. In this article the author gives references to other papers on Wronskians by Peano and himself.

$\dagger$ Loc. cit., p. 144.
} 
dependent. With the aid of Theorem $\mathrm{V}$ it can be shown that a necessary and sufficient condition for the verification of the hypothesis of Bôcher's theorem is that $M_{k}\left(u_{1}, u_{2}, \ldots, u_{n}\right)$ be of constant rank $m<n$.

NORTHWESTERN UNIVIERSITY, May, 1906.

\section{SIGNIFICANCE OF THE TERM HYPERCOMPLEX NUMBER.}

BY PROFESSOR JAMES BYRNIE SHAW.

(Read before the Chicago Section of the American Mathematical Society, April 14, 1906.)

Essentially four definitions of quite different logical import have been given for the term hypercomplex number, or multiple number. The four things so defined differ considerably in their mathematico-philosophical meaning, and while two of them are in a way equivalent, neither of the others can be correlated with these two or with each other as equivalent. It is proposed to examine these four definitions rather critically.

\section{The n-tuple.}

The first definition I shall denominate the Dedekind definition, although Hamilton discussed, many years before, entities defined in the same way. It is of a pure arithmetical character, since it implies only the existence of a set of things we may call numbers, marks, or entities, according as we conceive them to belong to a domain of integrity, an abstract field, or, in general, an aggregate that we can call a range. At first these entities were in a scalar domain, then they were generalized to a rational domain, then to an abstract field, and obviously we may take them from any range. The definition runs substantially thus : *

A set of $n$ ordered marks (entities) $a_{1}, \ldots, a_{n}$ of a field (range) $F$, is called an $n$-tuple element $a$. The symbol $a=$ $\left(a_{1}, \cdots, a_{n}\right)$ employed is purely positional without functional connotation. Its definition implies that $a=b$ if, and only if, $a_{1}=b_{1}, \cdots, a_{n}=b_{n}$.

* Dickson, "O)n hypercomplex number systems"; Transactions Amer. Math. Society, vol. 6 (1905), pp. 344-348. 